INDO GLOBAL JOURNAL OF

PHARMACEUTICAL SCIENCES

ISSN 2249- 1023

\title{
Ribosomal Maturation Factor RimP as a Potential Drug Target against $M$. fortuitum
}

\author{
Poonam, Jitendra Vashistt, Gopal Singh Bisht, Rahul Srivastava * \\ Department of Bioetchnology and Bioinformatics, Jaypee University of Information Technology, Waknaghat, Solan-173234 (H.P), India
}

Address for Correspondence: Rahul Srivastava, juit.rahul@gmail.com

Keywords RimP; M. fortuitum;

Antibiotic

Resistance; Drug

Target.

\begin{abstract}
Mycobacterium fortuitum is a non tuberculous, rapidly growing, acid fast and ubiquitous emergent pathogen attaining relevance in human health as it causes various clinical diseases ranging from localized cutaneous, bone, ocular and post-surgical infections to disseminated diseases. The pathogenesis of M. fortuitum is not clearly known, but it resides and multiplies inside macrophages, similar to other mycobacterial species. Aim of our study was to identify the membrane genes responsible for virulence of $M$. fortuitum to have an insight into its pathogenesis. As the genome sequence of $M$. fortuitum is not known, a transposon mutant library of 5000 mutants was constructed through random mutagenesis by electroporation of transposon TnphoA containing plasmid pRT291 into wild type M. fortuitum. Among 5000 mutants obtained, 127 blue mutants were confirmed for insertion of transposon in membrane gene by alkaline phosphatase activity. The mutants having highest alkaline phosphatase activity were further analyzed for their virulence attenuation in vivo by intravenous infection of mutants in female BALB/c mice followed by determining CFU of homogenized kidneys 10 days and 25 days post infection and antibiotic resistance profile. One mutant was found to be 4 fold sensitive against amikacin as compared to wild type, however no attenuation was observed in its virulence in vivo. The genomic analysis of the mutant was done by cloning of the inactivated membrane gene in pUC19 vector followed by sequencing. Bioinformatics analysis of the sequenced gene identified the gene as ribosomal maturation factor RimP showing 74\% amino acid homology and 50\% homology with Paenibacillus species and Mycobacterium abcessus respectively. Present study reveals the role of gene ribosomal maturation factor RimP in the antibiotic resistance of $M$. fortuitum that can be used as an important drug target against $M$. fortuitum. @ 2016 iGlobal Research and Publishing Foundation. All rights reserved.
\end{abstract}

Conference Proceedings: International Conference on Advances in Plant and Microbial Biotechnology (PMB2017); JIIT, Noida: February 02-04, 2017

Indo Global Journal of Pharmaceutical Sciences( ISSN 22491023 ; CODEN- IGJPAI; NLM ID: 101610675) indexed and abstracted in EMBASE(Elsevier), SCIRUS(Elsevier),CABI, CAB Abstracts, Chemical Abstract Services(CAS), American Chemical Society(ACS), Index Copernicus, EBSCO, DOAJ, Google Scholar and many more. For further details, visit http://iglobaljournal.com 